\title{
Synaptophysin Expression in Rat Retina Following Acute High Intraocular Pressure
}

\author{
Chen Dan ${ }^{1}$, Tong Jian-Bin ${ }^{1}$, Wang Hui ${ }^{1}$, Zeng Le-Ping ${ }^{1}$, Zhou Jin ${ }^{1}$, Huang Ju-Fang ${ }^{1}$ \\ and Luo Xue-Gang ${ }^{1}$
}

${ }^{1}$ Department of Anatomy \& Neurobiology, Xiangya School of Medicine, Central South University, Changsha, Hunan, 410013, China

Received September 23, 2008; accepted November 11, 2008; published online December 19, 2008

\begin{abstract}
In response to injury, synapse alteration may occur earlier than the changes in the cell body of neurons. Although retinal ganglion cell death and thinning of the inner part of retina were found after acute high intraocular pressure (HIOP), the structural and functional changes of synapses in the retina remain unknown. In the present study, we investigated the protein and mRNA expression of synaptophysin (SYN), an important molecule closely related to synaptic activities, synaptogenesis and synaptic plasticity. In addition, we also studied the ultrastructural changes of the retinal synapses. We found that (1) synaptophysin was upregulated transiently at both protein and mRNA level following HIOP; (2) broadened distribution of synaptophysin protein was present within the outer nuclear layer at the early stage following HIOP; (3) in the outer nuclear layer bouton-like vesicle-containing structures were observed by electron microscopy. This data suggested that, besides degeneration, synapses in rat retina may undergo regenerative events following HIOP.
\end{abstract}

Key words: synaptophysin, synapse, plasticity, degeneration, acute high intraocular pressure

\section{Introduction}

In rat retina, synapses in the outer and inner plexiform layers (OPL and IPL) play an important role in visual signal transmission. The synapses in the OPL are formed by terminals of photoreceptors with dendrites of bipolar cells and processes of the horizontal cells, and in the IPL, by axons of bipolar cells with dendrites of retinal ganglion cells (RGCs) and amacrine cell processes. Visual function is affected by changes of synaptic number or structure in these two layers [7]. Retinal injury may cause synapse alteration which occurs earlier than changes in cell body of neurons [4, 12]. Previous studies showed that acute high intraocular pressure (HIOP) caused thinning of the inner part of retina and loss of cells in ganglion cell layer (GCL) and inner nuclear layer (INL) $[2,6,10,11]$, while the structural and functional changes of synapses in the retina remain unknown.

Synaptophysin (SYN) is an integral membrane protein

Correspondence to: Huang Ju-Fang and Luo Xue-Gang, Department of Anatomy \& Neurobiology, Xiangya School of Medicine, Central South University, Changsha, 410013, China.

E-mail: jufanghuang@yahoo.com.cn (Huang Ju-Fang); xgluo@xysm.net (Luo Xue-Gang) of the synaptic vesicles. It possibly serves multiple functions in synaptic vesicle formation and exocytosis, playing an important role in neurotransmitter delivery. It is widely used as one of the synaptic function markers and is also thought to be closely related to synaptogenesis and synaptic plasticity during neural tissue development $[3,13]$. In the rat retina, SYN protein is expressed in a punctate pattern from postnatal day 4 and 12 onwards in the OPL and IPL, respectively, which is well correlated with the development of ribbon synapses. The expression pattern of SYN in retina indicated an important role of SYN in ribbon synapse formation and visual signal transmission [3].

In the present study, SYN was used to mark synapses. We investigated the protein and mRNA expression of SYN by immunohistochemistry and in situ hybridization, respectively. Our data showed that at the early stage following HIOP, SYN expression was transiently upregulated both at the protein and mRNA level in the ONL where there was no SYN immunostaining in normal condition. Based on these results, the ultrastructural changes in the outer nuclear layer (ONL) were further observed. Some bouton-like vesiclecontaining structures in ONL were revealed by electron microscopy. 


\section{Material and Methods}

\section{Animals}

Healthy adult Sprague-Dawley rats (200-250 g) used in the present study were given tap water and food in a light and temperature controlled room. All protocols for animal use were in accordance with the Guidelines laid down by the NIH. Following the procedure described by Adachi et al. [1], the animal model was prepared as follows. Animals were anesthetized with $2 \%$ pentobarbital $(40 \mathrm{mg} / \mathrm{kg})$. A drop of chloramphenicol eye drop was administered to the left conjunctiva sac. A 30 gauge needle connected with instillation instrument filling with normal saline was punctured into the anterior chamber through the lateral border of the left eye. The intraocular pressure was elevated to $110 \mathrm{mmHg}$ and maintained for $60 \mathrm{~min}$, then slowly descended to the normal level. Animals were allowed to survive for 1, 3, 7 or 14 days. For sham operation group, the needle was inserted into the anterior chamber without elevating the pressure. Each group was composed of 11 animals (4 for immunohistochemistry, 4 for in situ hybridization and 3 for electron microscopy). The right eyes served as normal control.

\section{Tissue preparation}

For immunohistochemistry and in situ hybridization, animals were anesthetized with $2 \%$ pentobarbital $(40 \mathrm{mg} / \mathrm{kg})$ and perfused transcardially with $0.9 \%$ sodium chloride, followed by $4 \%$ paraformaldehyde in phosphate buffer $(0.1 \mathrm{M}$, $\mathrm{pH}$ 7.4). After perfusion, the eyeballs were dissected out, the cornea and lenses removed and post-fixed in the same fixative for $2 \mathrm{hr}$ at room temperature, then dehydrated by immersed gradually in $15 \%$ and $30 \%$ sucrose phosphate buffer (for immunohistochemistry) or kept overnight in sterile 30\% sucrose phosphate buffer (for in situ hybridization) at $4^{\circ} \mathrm{C}$. Cryosections (20 $\mu \mathrm{m}$ thickness) were cut with microtome and the sections with optic nerve were selected and stored at $-20^{\circ} \mathrm{C}$ prior to processing. To minimize methodological variations, control and the experimental sections of each time point were placed on the same slide. For electron microscopy, animals were anesthetized with $2 \%$ pentobarbital $(40 \mathrm{mg} / \mathrm{kg}$ ) and the eyeballs were dissected out freshly, cornea, lenses and sclera removed and the inferior temporal quadrant were selected and fixed with $1.2 \%$ paraformaldehyde $/ 0.8 \%$ glutaraldehyde at $4{ }^{\circ} \mathrm{C}$ overnight. The animals were sacrificed by cervical dislocation.

\section{Immunohistochemistry}

Sections were soaked for $15 \mathrm{~min}$ in $0.3 \% \mathrm{H}_{2} \mathrm{O}_{2}$ in PBS to inactivate endogenous peroxidase. After the elimination of non-specific antibody binding in 5\% bovine serum in PBS containing $0.05 \%$ TritonX-100, the sections were incubated with a mouse SYN antibody (Chemicon, Temecula, CA) at a dilution of $1: 1000$ at $4{ }^{\circ} \mathrm{C}$ overnight, then with a biotinylated anti-mouse antibody at a dilution of 1:200 (Vector Laboratories Inc., Burlingame, $\mathrm{CA}$ ) for $1 \mathrm{hr}$ at room temperature. After incubation with avidin-biotin complex (ABC, Vector Laboratories Inc., Burlingame, CA) for $1 \mathrm{hr}$ at room temper- ature, the sections were visualized following incubation in a PBS solution containing $0.05 \%$ DAB and $0.03 \% \mathrm{H}_{2} \mathrm{O}_{2}$ for 5 min at room temperature. Finally, the sections were dehydrated, cleared and coverslipped. In order to exclude false positive results, control sections were processed in exactly the same way except for the replacement of the primary antibody by PBS.

\section{In situ hybridization}

Hybridization procedures were carried out according to the instructions provided by the SYN in situ hybridization kit (Boshide, China). In brief, the sections were treated with $0.6 \% \mathrm{H}_{2} \mathrm{O}_{2}$ (diluted in pure methanol) for $30 \mathrm{~min}$ to inactivate the endogenous peroxidase. Fragments of mRNA nucleic acid were exposed by treatments with pepsin (diluted by $3 \%$ citric acid) for $1 \mathrm{~min}$. Sections were post-fixed with $1 \%$ paraformaldehyde for $10 \mathrm{~min}$ at room temperature. After washing thrice in sterile $0.01 \mathrm{M}$ PBS for $5 \mathrm{~min}$, prehybridization was carried out with prehybridization buffer at $42^{\circ} \mathrm{C}$ for $3 \mathrm{hr}$. The sections were then hybridized with the digoxin conjugated SYN cDNA probes (the sequences of the mRNA to the target gene fragments are: 5-TCAGT TCCGG GTGGT CAAGG AGCCC CTTGG CTTCG-3; 5-CACTA CCAAG ATCTT CCTGG TTGGG GACTA CTCCT-3; 5TGCCC ATGTG CCGCC AGACA GGGAA CACAT GCAAG-3) in a humid chamber at $42^{\circ} \mathrm{C}$ overnight. After the sections were washed at $37^{\circ} \mathrm{C}$ twice with $2 \times \mathrm{SSC}(17.53 \mathrm{~g}$ sodium chloride and $8.82 \mathrm{~g}$ sodium citrate in $1000 \mathrm{ml}$ distilled water, $\mathrm{pH} 7.0$ ) for $5 \mathrm{~min}$, once with $0.5 \times \mathrm{SSC}$ for 15 min and once with $0.2 \times \mathrm{SSC}$ for $15 \mathrm{~min}$, the sections were incubated in blocking buffer at $37^{\circ} \mathrm{C}$ for $30 \mathrm{~min}$, biotin conjugated anti-mouse digoxin at room temperature for $2 \mathrm{hr}$, streptavidin-biotin complex (SABC) $30 \mathrm{~min}$ and biotin conjugated peroxidase for $30 \mathrm{~min}$. The last three steps were separated by thrice washing with PBS at room temperature for 5 $\min$. Sections were colorized by incubation with a PBS solution containing $0.05 \% \mathrm{DAB}$ and $0.03 \% \mathrm{H}_{2} \mathrm{O}_{2}$ for $5 \mathrm{~min}$ at room temperature. Finally, the sections were dehydrated through graded ethanol $(70 \%, 85 \%, 95 \%$ and $100 \%)$, cleared in xylene, and coverslipped. Controls were set to ensure the specificity of the probe and the SABC system, including RNase treatment of sections before hybridization with the probe and hybridization with PBS instead of the probe, respectively.

\section{Electron microscopy}

For electron microscopy, after washing in $0.1 \mathrm{M} \mathrm{PB}$, the tissues were post-fixed in $1 \%$ osmium tetroxide for 1.5 $\mathrm{hr}$, and then washed with distilled water. The tissues were pre-stained with $2 \%$ aqueous uranyl acetate for $30 \mathrm{~min}$, and then dehydrated gradually and embedded with ProcureAraldite. Ultrathin sections $(90 \mathrm{~nm})$ were cut with ultramicrotome (Leica, Germany). Sections on grids were observed under electron microscope (JEOL, Japan).

\section{Data analysis}

Six sections were selected from each retina. Under $40 \times$ 
objective of Motic image system (Motic, China), four regions symmetrical to the optic nerve (two consecutive regions of each side) were chosen from each section for photo taking. Pictures were analyzed with Motic Images Advanced 3.2 system. In this system, average gray value from black to white ranged from 0 to 255 . In the present study, we calculated the parameter "gray value" (difference between average gray value of the background and that of the positive products) in the IPL, OPL and ONL for immunohistochemistry and in the ONL and INL for in situ hybridization. We chose the non-tissue area inner to the GCL as background reference area. For immunohistochemistry, the "area of positive products" was also surveyed in the OPL, ONL and IPL. The parameter "total amount of staining" was defined as the "gray value" times the "area of positive products". "Total amount of staining" was used to gain insight into the total staining signal independent of changes of protein redistribution and tissue swelling or shrinkage [4]. The values of the experimental retinae were compared with those of the control retinae $(=100 \%)$ present on the same slide [4]. Statistical analysis was done by one way-ANOVA with SPSS13.0 software, with $\mathrm{p}$-values $<0.05$ being considered significant.

\section{Results}

In normal rats, SYN immunoreactivity was found in the OPL and IPL (Fig. 1B, H). After HIOP, besides the above two layers, SYN immunoreactivity was also found in the inner part of the ONL and reached the maximum "area of positive products" in OPL and ONL at the 7th day post injury (Fig. 1B-E, H-K). Thus, though "gray value" was just slightly increased in OPL and ONL after injury, the "total amount of staining" in ONL and OPL showed significant increase of SYN expression at the 3rd and 7th day compared to normal level (Fig. 1M). At the 14th day, SYN distribution in the OPL and ONL returned to normal pattern (Fig. 1F, L,
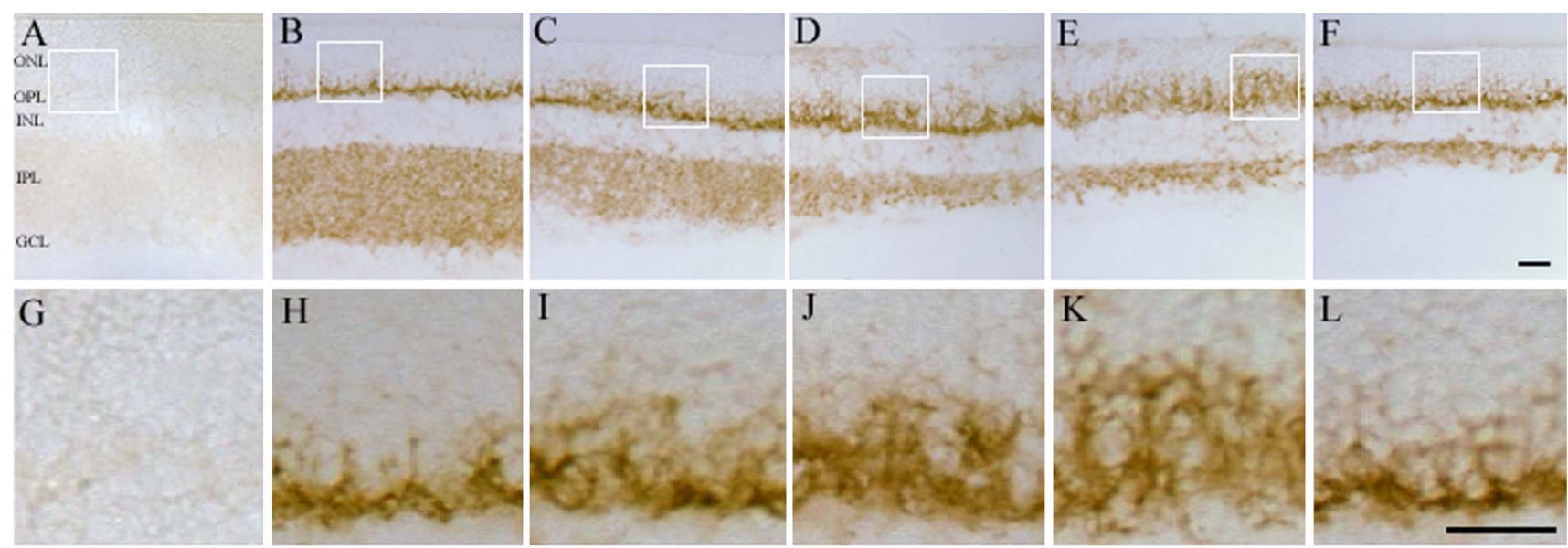

M

$\mathrm{N}$
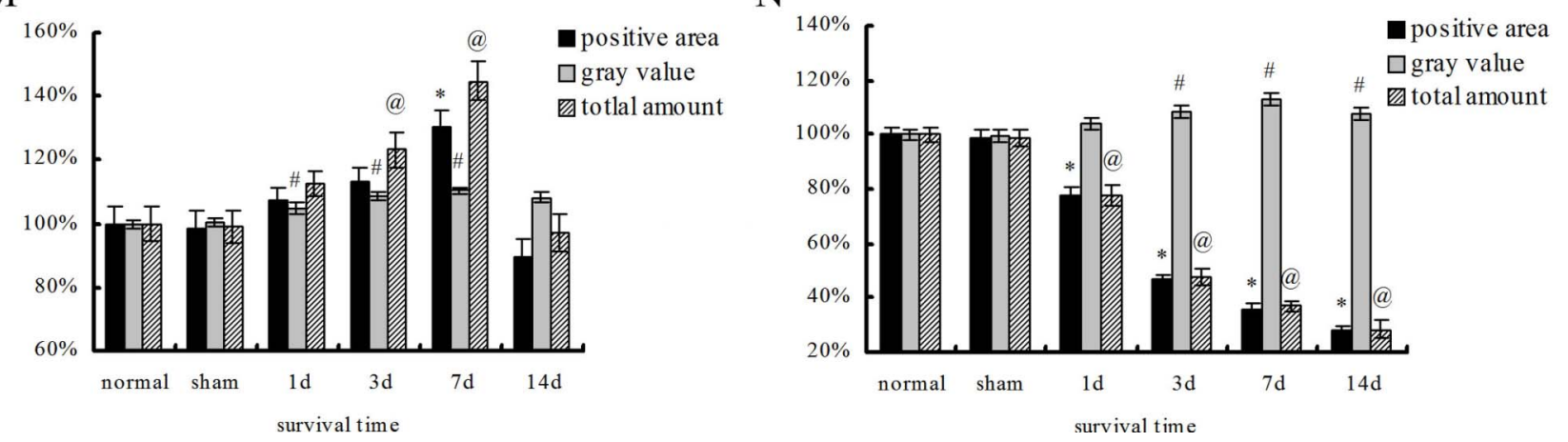

Fig. 1. Light micrographs of immunohistochemistry of synaptophysin in rat retina at different survival time points after acute HIOP. Bar=20 $\mu \mathrm{m}$. Negative control (A, G), normal control $(\mathbf{B}, \mathbf{H}), 1$ st day $(\mathbf{C}, \mathbf{I})$, 3rd day $(\mathbf{D}, \mathbf{J}), 7$ th day $(\mathbf{E}, \mathbf{K})$ and 14th day $(\mathbf{F}, \mathbf{L})$ following HIOP. G-L were the higher magnification of A-F. ONL, outer nuclear layer; OPL, outer plexiform layer; INL, inner nuclear layer; IPL, inner plexiform layer; GCL, ganglion cell layer. SYN positive products were expanded in the OPL and the inner part of the ONL and reached the maximum at the 7th day after HIOP $(\mathbf{E}, \mathbf{K}, \mathbf{M})$ and returned to normal level at the 14th day $(\mathbf{B}, \mathbf{H}, \mathbf{F}, \mathbf{L}, \mathbf{M})$. Gray value of SYN in the OPL and ONL was slightly elevated from the 1st day to the 7th day, then returned to normal level (M). Total amount of staining showed significant increase of SYN expression at the 3rd and 7th day comparing to normal level (M). It showed a slight increase of gray value of SYN in the IPL at the 3rd, 7th and 14th day after HIOP. But with marked thinning of the IPL induced by HIOP injury, the total amount of staining decreased dramatically (N). M: Analysis of SYN immunoreactivity in the OPL and ONL in rat retina, N: Analysis of SYN immunoreactivity in the IPL of rat retina. *, \# and @ $P<0.05$ vs normal control group. 


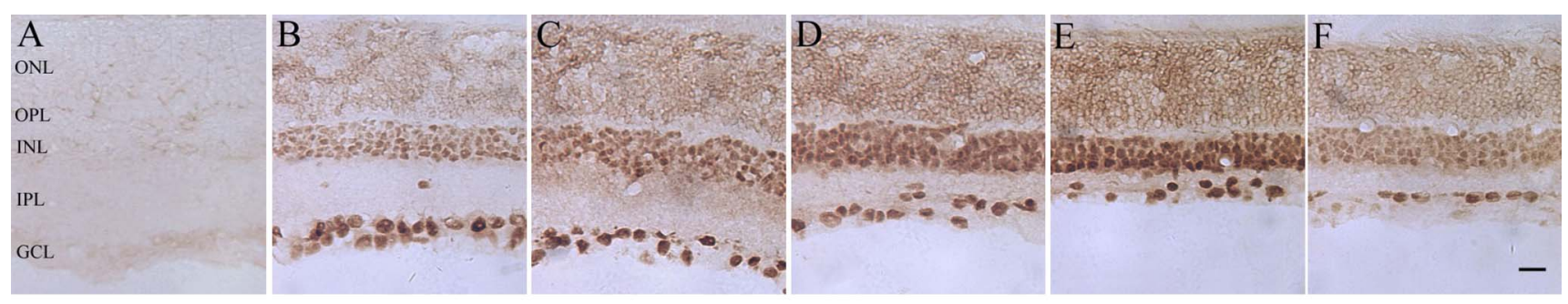

G

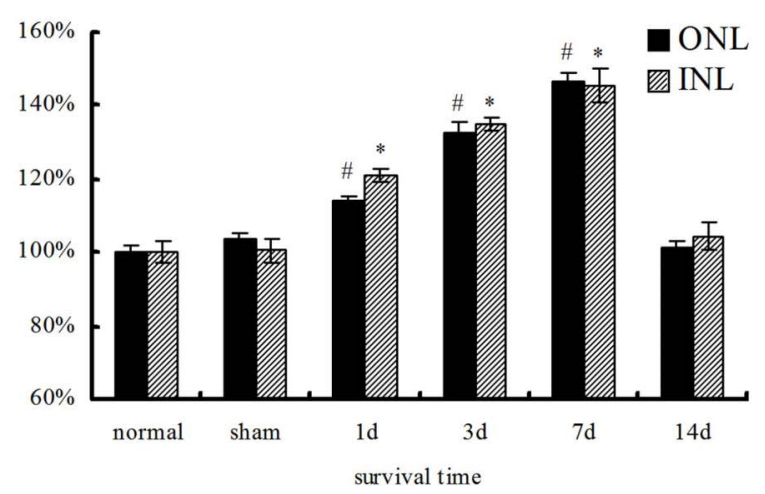

Fig. 2. Light micrographs of in situ hybridization of synaptophysin mRNA in rat retina at different survival time points after acute HIOP. Bar=20 $\mu \mathrm{m}$. Negative control (A), normal control $(\mathbf{B})$, 1st day $(\mathbf{C})$, 3rd day (D), 7th day $(\mathbf{E})$ and 14th day (F) following HIOP. ONL, outer nuclear layer; OPL, outer plexiform layer; INL, inner nuclear layer; IPL, inner plexiform layer; GCL, ganglion cell layer. The amount of SYN mRNA positive products in ONL and INL were increased $(\mathbf{C}, \mathbf{D}, \mathbf{E}, \mathbf{G})$ at the $1 \mathrm{st}, 3 \mathrm{rd}$ and 7 th day after HIOP and reverted to normal level at the 14th day, $(\mathbf{B}, \mathbf{F}, \mathbf{G})$. G: Analysis of the gray value of SYN mRNA positive products in the ONL and INL of rat retina. *, $P<0.05$ vs normal control group.

M). SYN immunoreactivity was also found in IPL and it also showed a slight increase of gray value at the $3 \mathrm{rd}, 7$ th and 14th day after HIOP. But with the marked thinning of the IPL induced by HIOP injury, the total amount of staining was decreased dramatically (Fig. 1B-F, N).

Furthermore, to investigate SYN expression changes from transcription level, in situ hybridization was employed for SYN mRNA detection. SYN mRNA was found in each nuclear layer in normal rat retina (Fig. 2B). The gray value of ONL and INL gradually increased 1 day, 3 days and 7 days after HIOP (Fig. 2C-E, G), indicating an increased expression of SYN mRNA. At the 14th day, the gray value reverted to normal level (Fig. 2F, G). In GCL, the number of SYN mRNA positive cells was dramatically decreased due to the loss of neurons in GCL after HIOP.

It was interesting to note that a broadened distribution of SYN protein was found in the ONL after HIOP since, in normal condition, there is no SYN immunostaining in this layer. Furthermore, we observed the ultrastructure changes in the ONL. It was found that at the 3rd and 7th day after HIOP, there were some bouton-like vesicle-containing structures found in the ONL at least 4 rows of nuclei away from the OPL, where synapses and bouton-like vesiclecontaining processes could not be found in normal condition (Fig. 3). This finding may be coincident with the broadened protein expression of SYN in the ONL at the early stage following HIOP. No typical synaptic structures were found in the ONL.

\section{Discussion}

In the present study, the expression of SYN, an important protein associated with synaptic function, was investigated in rat retina following HIOP. It was found that SYN protein was not only present in the OPL and IPL, but also present and increased in the inner part of the ONL at the early stage following HIOP. Forty percent increase of the total amount of staining was detected as compared to normal level. SYN mRNA was also found upregulated along the same time course as the enlargement of the distribution area of SYN protein, indicating that the increased SYN protein in OPL and ONL was due to increased gene transcription. The increased SYN in ONL may suggest synaptic plasticity in rat retina following HIOP. Interestingly, 3 and 7 days after HIOP, some bouton-like vesicle-containing structures were found ectopic distribution with the electron microscopy in the ONL, where SYN immunoreactivity was absent in normal rat retina. The findings in ONL by electron microscopy may be related to the broadened distribution of SYN in ONL after HIOP injury. However, to clarify this issue further experiments are needed. Why SYN was increased in ONL is not known. It was likely that the increased synthesized protein was being transported to the terminal of the photoreceptors. It may also imply new processes sprouting from the photoreceptors or horizontal cells. Further studies would be needed to clarify this phenomenon. Because of cell death in GCL and INL following HIOP [2, 6, 10, 11], synapses 

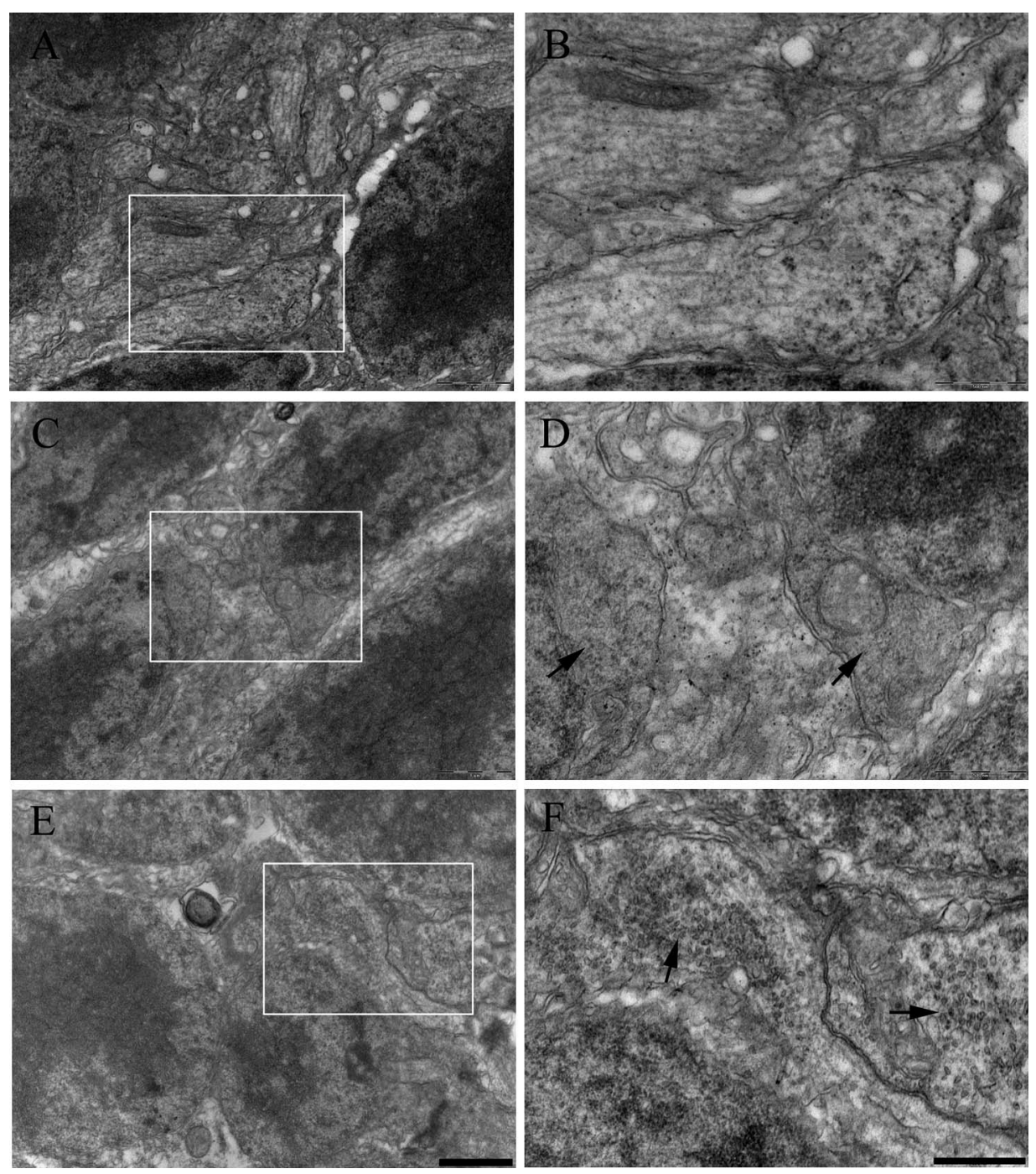

Fig. 3. Bouton-like vesicle-containing structures found in the ONL with electron microscopy at the 3rd and 7th day following HIOP injury. In normal condition (A, B), no synapses and vesicle containing processes could be found. Some bouton-like vesicle-containing structures were found in the ONL at least 4 rows of nuclei away from the OPL 3 and 7 days after HIOP injury $(\mathbf{C}, \mathbf{D}, \mathbf{E}, \mathbf{F})$. B, D and $\mathbf{F}(\mathrm{Bar}=500 \mathrm{~nm})$ were higher magnification of the selected parts in $\mathbf{A}, \mathbf{C}$ and $\mathbf{E}$ (Bar=1 $\mu \mathrm{m})$. Arrows show vesicles.

between RGCs, bipolar and amacrine cells underwent degenerative processes. The thickness of IPL decreased markedly after HIOP. By the 14th day, the positive protein area of SYN reduced to about $70 \%$. The total amount of SYN protein was reduced along with the decreasing thickness of IPL despite the increase of gene transcription in neurons in the INL.

Dijk et al. recently reported that GAP-43 expression was transiently upregulated in the GCL and IPL after HIOP injury, indicating regenerative processes involved in the remodeling of the retinal network after retina injury [4]. In the present study, it was found that the SYN expression was also enhanced at the early stage following HIOP injury, and interestingly, SYN distribution was enlarged in the OPL and ONL, where there were no significant changes in cell number, thickness and expression of proteins sensitive to injury after HIOP $[2,10,11]$. Although caspase 3 was once reported to be upregulated transiently in the ONL after HIOP, the upregulation occurred immediately after HIOP and only lasted for several hours, and returned to normal level within $24 \mathrm{hr}$ [5]. It was unlikely that the direct response to HIOP of the neurons in ONL induced the change of expression of SYN several days later after injury. It was reported that, besides the damage to the RGCs, the neurons in the lateral geniculate bodies may undergo antegrade trans-synaptic changes following HIOP $[5,14]$. Then whether there were retrograde trans-synaptic changes within retina after HIOP? During the early stage of the injury induced by HIOP, visual signal transmission was partly blocked from RGCs to neurons in the lateral geniculate bodies due to severe damage of RGCs $[1,8,9]$. As a compensatory mechanism, the loss of signal transmission may enhance the activity of the remain- 
ing circuits of visual signal transmission upstream to RGCs, and may lead to increased production of proteins related to synaptic activities, such as SYN and generation of new neuronal processes for new synapse formation. Thus, after HIOP injury, synapses on the visual signal pathway upstream to RGCs may undergo some retrograde trans-synaptic plasticity. More work is needed to clarify the significance and mechanisms involved in this transient upregulation and redistribution of SYN after HIOP.

In conclusion, SYN was transiently upregulated with broadened distribution in rat retina following HIOP injury, which indicated that, besides degeneration, synapses in rat retina may undergo regenerative events following HIOP injury.

\section{Acknowledgements}

This work was supported by the National Natural Science Foundation of China (30570979, 30671100) and the Hunan Provincial Natural Science Foundation of China (08JJ5042).

\section{References}

1. Adachi, M., Takahashi, K., Nishikawa, M., Miki, H. and Uyama M. (1996) High intraocular pressure-induced ischemia and reperfusion injury in the optic nerve and retina in rats. Graefes Arch. Clin. Exp. Ophthalmol. 234; 445-451.

2. Cheon, E. W., Park, C. H., Kang, S. S., Cho, G. J., Yoo, J. M., Song, J. K. and Choi, W. S. (2003) Betaxolol attenuates retinal ischemia/reperfusion damage in the rat. Neuroreport $14 ; 1913$ 1917.

3. Dhingra, N. K., Ramamohan, Y. and Raju, T. R. (1997) Developmental expression of synaptophysin, synapsin I and syntaxin in the rat retina. Brain Res. Dev. Brain Res. 102; 267-273.

4. Dijk, F., Bergen, A. A. and Kamphuis, W. (2007) GAP-43 expression is upregulated in retinal ganglion cells after ischemia/reperfusion-induced damage. Exp. Eye Res. 84; 858-867.

5. Gupta, N., Ly, T., Zhang, Q., Kaufman, P. L., Weinreb, R. N. and Yucel, Y. H. (2007) Chronic ocular hypertension induces dendrite pathology in the lateral geniculate nucleus of the brain. Exp. Eye Res. 84; 176-184.

6. Huang, J. F., Xiong, K., Tong, J. B., Chen, D., Pan, A. H. and Luo, X. G. (2005) Changes of inner retina under different ischemic reperfusion induced by acute intraocular hypertension in rats. Chin. J. Anat. 28; 46-49.

7. Nag, T. C. and Wadhwa, S. (2001) Differential expression of syntaxin-1 and synaptophysin in the developing and adult human retina. J. Biosci. 26; 179-191.

8. Nucci, C., Gasperi, V., Tartaglione, R., Cerulli, A., Terrinoni, A., Bari, M., De Simone, C., Agro, A. F., Morrone, L. A., Corasaniti, M. T., Bagetta, G. and Maccarrone, M. (2007) Involvement of the endocannabinoid system in retinal damage after high intraocular pressure-induced ischemia in rats. Invest. Ophthalmol. Vis. Sci. 48; 2997-3004.

9. Nucci, C., Tartaglione, R., Cerulli, A., Mancino, R., Spano, A., Cavaliere, F., Rombola, L., Bagetta, G., Corasaniti, M. T. and Morrone, L. A. (2007) Retinal damage caused by high intraocular pressure-induced transient ischemia is prevented by coenzyme Q10 in rat. Int. Rev. Neurobiol. 82; 397-406.

10. Sakamoto, K., Yonoki, Y., Kuwagata, M., Saito, M., Nakahara, T. and Ishii, K. (2004) Histological protection against ischemiareperfusion injury by early ischemic preconditioning in rat retina. Brain Res. 1015; 154-160.

11. Singh, M., Savitz, S. I., Hoque, R., Gupta, G., Roth, S., Rosenbaum, P. S. and Rosenbaum, D. M. (2001) Cell-specific caspase expression by different neuronal phenotypes in transient retinal ischemia. J. Neurochem. 77; 466-475.

12. Tropea, D., Caleo, M. and Maffei, L. (2003) Synergistic effects of brain-derived neurotrophic factor and chondroitinase $\mathrm{ABC}$ on retinal fiber sprouting after denervation of the superior colliculus in adult rats. J. Neurosci. 23; 7034-7044.

13. Valtorta, F., Pennuto, M., Bonanomi, D. and Benfenati, F. (2004) Synaptophysin: leading actor or walk-on role in synaptic vesicle exocytosis? Bioessays 26; 445-453.

14. Yucel, Y. H., Gupta, N., Zhang, Q., Mizisin, A. P., Kalichman, M. W. and Weinreb, R. N. (2006) Memantine protects neurons from shrinkage in the lateral geniculate nucleus in experimental glaucoma. Arch. Ophthalmol. 124; 217-225.

This is an open access article distributed under the Creative Commons Attribution License, which permits unrestricted use, distribution, and reproduction in any medium, provided the original work is properly cited. 